\title{
ACESSIBILIDADE DA ESTRUTURA FÍSICA HOSPITALAR PARA A PRÁTICA DA HIGIENIZAÇÃO DAS MÃOS
}

\author{
Accessibility of the infrastructure for the hospital practice of hand hygiene \\ Accesibilidad de la estructura física para la práctica hospitalaria de higiene de las \\ manos
}

\section{RESUMO}

A higienização das mãos é o procedimento mais simples e eficaz na prevenção e controle das infecções relacionadas à assistência à saúde. Contudo, a adesão a esta prática é excessivamente baixa. Considera-se a infraestrutura inadequada dos serviços de saúde um dos fatores relacionados à baixa adesão. Diante disso, conduziu-se um estudo observacional e transversal para avaliar a infraestrutura hospitalar para a prática da higienização das mãos. Os sujeitos e a fonte de informação foram médicos, enfermeiros e a estrutura das unidades de um hospital da região noroeste do Paraná. Utilizou-se para a coleta de dados um instrumento estruturado e, para a análise, a estatística descritiva. Os insumos e equipamentos, tais como pias e dispensadores para a prática da higienização das mãos, eram limitados. Existem falhas na infraestrutura para a prática da higienização das mãos, o que pode comprometer a qualidade da assistência e a segurança do paciente.

Palavras-chave: Lavagem das mãos. Serviços de saúde. Estrutura dos serviços. Infecção hospitalar.

\begin{abstract}
Hand hygiene is the simplest and effective in the prevention and control of infections related to health care. However, adherence to this practice is too low. It is considered the inadequate infrastructure of health services as one of the factors related to poor adherence. Therefore, we conducted a cross-sectional observational study to assess the hospital infrastructure, for the practice of hand hygiene. Subjects and source of information were physicians, nurses and structure of units of a hospital in northwestern Paraná. It was used for data collection and a structured instrument for the analysis, descriptive statistics. The supplies and equipment such as sinks and dispensers for the practice of hand hygiene was limited. There are gaps in infrastructure for the practice of hand hygiene which can compromise the quality of care and patient safety.
\end{abstract}

Keywords: Handwashing. Health Services. Structure of Services. Cross Infection.

\section{Resumen}

La higiene de las manos es el procedimiento más simples y eficaz en la prevención y control de las infecciones relacionadas con el cuidado de la salud. Sin embargo, la adhesión a esta práctica es muy baja. Se considera la infraestructura inadecuada de los servicios de salud como uno de los factores relacionados con la falta de adherencia. Por lo tanto, se realizó un estudio observacional transversal para evaluar la infraestructura hospitalaria para la práctica de la higiene de las manos. Los sujetos y las fuentes de información eran médicos, enfermeras y la estructura de las unidades de un hospital en el noroeste de Paraná. Se utilizó, para la recolección de datos, un instrumento estructurado y, para el análisis, la estadística descriptiva. Los suministros y equipamientos, tales como lavabos y dispensadores para la práctica de la higiene de las manos era limitada. Hay deficiencias en la infraestructura para la práctica de la higiene de las manos que pueden comprometer la calidad de la atención y la seguridad del paciente.

Palabras clave: Lavado de manos. Servicios de Salud. Estructura de los Servicios. Infección Hospitalaria.

'Mestre em Ciências da Saúde pela Universidade Estadual de Maringá-PR. Docente do Curso de Enfermagem da Universidade Estadual do Paraná. Campus de Paranavaí-PR. Brasil. E-mail: mfpprado@gmail.com; ${ }^{2}$ Acadêmica do Curso de Enfermagem do Centro Universitário de Maringá-PR. Brasil. E-mail: enftalitaps@yahoo.com.br; ${ }^{3}$ Acadêmico do Curso de Medicina da Universidade do Oeste Paulista de Presidente Prudente-SP. Brasil. E-mail: beto_1202@hotmail.com. 


\section{INTRODUÇÃO}

A higienização das mãos é um importante indicador de qualidade dos serviços de saúde para a segurança do paciente', pois é considerada a medida individual mais simples e eficaz para prevenir e controlar as infecções relacionadas à assistência à saúde e a disseminação de microrganismos multirresistentes².

No entanto, a adesão a esta prática pelos profissionais da saúde é inaceitavelmente baixa ${ }^{1,2}$. São multifatoriais as barreiras relacionadas à baixa adesão. No aspecto institucional, abrangem a ausência de manuais nos setores, a carência de produtos adequados para a higienização das mãos, a escassez de pias e dispensadores ou a sua má localização, a falta de cultura institucional para a prática da higienização das mãos e ausência de liderança administrativa para estimular a adesão ou punir aqueles que a negligenciam ${ }^{3}$.

Apesar de as evidências científicas mostrarem a relação entre a acessibilidade às pias e dispensadores e 0 aumento significativo na taxa de adesão à higienização das mãos pelos profissionais da saúde $e^{4,5}$, ainda existem falhas na oferta de insumos e equipamentos nos serviços de saúde, tais como a dificuldade de acesso às pias e dispensadores, localização distante do ponto da assistência ao paciente e instalação em pontos ergonomicamente incorretos, o que pode dificultar esta adesão $0^{6}$. A Organização Mundial da Saúde (OMS) destaca ainda que, se pias ou dispensadores com álcool não estiverem prontamente acessíveis e se a higienização das mãos não for realizada, potencializa-se o risco de infecção relacionada à assistência à saúde e, consequentemente, a morbidade, mortalidade e custos relacionados ${ }^{1}$.

Muitos estudos discutem a temática higienização das mãos; entretanto, no Brasil, a infraestrutura dos serviços de saúde voltada a esta prática é pouco investigada. Assim, o presente estudo objetivou avaliar a estrutura física para a prática da higienização das mãos em um serviço de assistência à saúde hospitalar.

\section{MÉTODOS}

Trata-se de um estudo descritivo, observacional e transversal conduzido em quinze unidades assistenciais de um serviço de assistência à saúde hospitalar da região noroeste do Paraná.

Os sujeitos da pesquisa foram os profissionais da saúde, incluindo 31 médicos, sendo um deles o coordenador da Comissão de Controle de Infecção Hospitalar (CCIH), e 13 enfermeiros responsáveis pelas unidades observadas. Como fonte de informação foi utilizada a estrutura física das 15 unidades assistenciais, entre o Berçário, Centro Cirúrgico, Clínica Médica/Cirúrgica (I), Clínica Médica/Cirúrgica (II), Clínica Médica (I), Clínica Médica (II), Clínica Médica (III), Diálise, Endoscopia, Obstetrícia, Pediatria, Quimioterapia, Unidade de Emergência, Unidade de Tratamento Intensivo (UTI) Adulto e Unidade de Terapia Intensiva (UTI) Pediátrica. Estabeleceram- se como critérios de inclusão: os médicos deveriam estar presentes nas unidades no momento das observações, os enfermeiros deveriam ser os responsáveis pelas unidades assistenciais e as unidades deveriam conter o ponto de assistência e tratamento, que é o local onde os três elementos ocorrem juntos: o paciente, o profissional de saúde e a assistência, envolvendo o contato com o paciente.

Realizou-se o estudo nos meses de julho e agosto de 2010. Para a coleta de dados selecionou-se um único entrevistador e observador, treinado em relação aos aspectos relacionados à observação direta para prevenir viés de informação e interferência na rotina do setor. Utilizouse para a coleta de dados um instrumento estruturado, publicado pela $\mathrm{OMS}^{7}$, contendo 28 questões direcionadas aos diferentes sujeitos e à estrutura física, com destaque às seguintes variáveis: 1) direcionadas ao coordenador da $\mathrm{CCIH}$ : periodicidade das auditorias sobre a adesão à higienização das mãos nas unidades; 2) direcionadas ao enfermeiro responsável pela unidade: existência de manuais sobre higienização das mãos nos setores e treinamentos recebidos sobre o tema nos últimos dois anos; 3 ) direcionadas aos médicos: treinamentos recebidos sobre 0 tema nos últimos dois anos; 4) referentes à estrutura física: disponibilidade da água e preparações alcoólicas, número de dispensadores e pias nos setores, tipos de dispensadores, localização dos dispensadores e pias, presença de cartazes de promoção à higienização das mãos dispostos próximos às pias e dispensadores e número de pias e dispensadores por leito.

Para a coleta de dados referentes à estrutura, houve a observação direta das unidades assistenciais, com respeito a não interferir na rotina dos profissionais da saúde e na assistência aos pacientes. Além disso, para a entrevista, foi explicado o propósito da pesquisa aos sujeitos e os dados foram coletados mediante a assinatura do Termo de Consentimento Livre e Esclarecido. Os resultados obtidos foram analisados quantitativamente por meio da estatística descritiva, expressos por distribuição de frequência absoluta e relativa e apresentados em tabelas. 0 estudo foi aprovado pelo Comitê de Ética em Pesquisa mediante o certificado $124 / 2010$.

\section{RESULTADOS}

Houve variação na disponibilidade de insumos e equipamentos para a prática da higienização das mãos, conforme demonstra a Tabela 1.

Em relação ao tipo de dispensadores disponíveis nas unidades assistenciais, predominou o tipo de dispensador de parede, totalizando 258 dispensadores. A maioria destes (211; $81,8 \%$ ) estava presente nos pontos de assistência e tratamento, no entanto, distante do alcance das mãos, e 47 (18,2\%) estavam localizados nos corredores. Somente um dispensador do tipo frasco afixado ao leito estava disponível na Unidade de 
Terapia Intensiva. Os dispensadores do tipo frasco de bolso e frasco afixado no carrinho ou bandeja não foram observados.

Nas unidades, constatou-se a escassez de cartazes ilustrativos sobre a técnica e sobre a promoção da higienização das mãos fixados nas unidades. Na maioria das unidades (8; $53,3 \%$ ) não havia manuais sobre a temática disponíveis para consulta pelos profissionais da saúde (Tabela 2).

Referente ao treinamento recebido pelos enfermeiros e médicos sobre a higienização das mãos, nos últimos dois anos, verificou-se a disparidade entre as categorias profissionais, pois a maioria dos enfermeiros (12; 92\%) receberam treinamento e apenas $1(8 \%)$ não recebeu. Entre os médicos, a maioria deles $(24 ; 80 \%)$ não recebeu e somente $6(20 \%)$ receberam treinamento.
Além disso, constatou-se que as auditorias sobre a higienização das mãos não eram realizadas pela CCIH nas unidades assistenciais.

Nas unidades assistenciais, verificou-se a taxa de pia por leitos, na metade destas $(8 ; 50,0 \%)$ as pias estavam disponíveis nos pontos de assistência e tratamento, com variação na taxa entre as unidades; a maior taxa (1:1) foi a da UTI adulto e a menor (1:6) foi a da Quimioterapia. Em relação ao número de dispensadores por leito, identificouse que na maioria das unidades $(10 ; 62,5 \%)$ não havia dispensadores nos pontos de assistência e tratamento, nas demais, houve discrepância na variação da taxa entre a maior (1:1) na UTI adulto e a menor (1:41) na Obstetrícia (Tabela 3).

Tabela 1 - Distribuição dos insumos e equipamentos para a prática da higienização das mãos, segundo a sua disponibilidade nas unidades assistenciais. Maringá, PR, 2010.

\begin{tabular}{|c|c|c|c|c|}
\hline \multirow{2}{*}{ Insumos/ Equipamentos } & \multicolumn{4}{|c|}{ Disponibilidade nas Unidades $(n=15)$} \\
\hline & Sempre (\%) & Esporadicamente (\%) & Raramente $(\%)$ & Nunca $(\%)$ \\
\hline Água Disponivel & $15(100)$ & - & - & - \\
\hline $\begin{array}{l}\text { Preparação alcoólica para } \\
\text { higienização das mãos } \\
\text { disponivel nos setores }\end{array}$ & $10(66,7)$ & $4(26,7)$ & $1(6,6)$ & - \\
\hline $\begin{array}{l}\text { Profissional de saúde tem fácil } \\
\text { acesso a frascos de bolso } \\
\text { preparação alcoólica }\end{array}$ & - & - & - & $15(100)$ \\
\hline $\begin{array}{l}\text { Dispensadores são abastecidos } \\
\text { quando vazios }\end{array}$ & $2(13,3)$ & $13(86,7)$ & - & - \\
\hline
\end{tabular}

Tabela 2 - Distribuição dos tipos de cartazes ilustrativos sobre higienização das mãos considerando a disponibilidade nas unidades assistenciais. Maringá, PR, 2010.

\begin{tabular}{lcc}
\hline \multicolumn{1}{c}{ Tipos } & \multicolumn{2}{c}{ Disponibilidade nas Unidades ( $\mathbf{n}=15)$} \\
\cline { 2 - 3 } & \multicolumn{1}{c}{ Sim (\%) } & Não (\%) \\
\hline $\begin{array}{l}\text { Técnica higienização simples das } \\
\text { mãos, expostos ao lado de cada pia }\end{array}$ & $1(6,6)$ & $14(93,4)$ \\
$\begin{array}{l}\text { Técnica friç̧ão antisséptica das } \\
\text { mãos, expostos no ponto de } \\
\text { assistência/tratamento }\end{array}$ & - & $15(100)$ \\
$\begin{array}{l}\text { Indicações para higienização das } \\
\text { mãos, expostos no ponto de } \\
\text { assistência/tratamento }\end{array}$ & - & $15(100)$ \\
$\begin{array}{l}\text { Cartazes de promoção à higienização } \\
\text { das mãos }\end{array}$ & $7(46,7)$ & $8(53,3)$ \\
$\begin{array}{l}\text { Manuais sobre as recomendações de } \\
\text { higienização das mãos }\end{array}$ & $7(46,7)$ & $8(53,3)$ \\
\hline
\end{tabular}


Higienização das mãos: acessibilidade da estrutura física

Tabela 3 - Distribuição da taxa de pia por leito e taxa de dispensadores com solução alcoólica afixados no leito e ao alcance das mãos. Maringá, PR, 2010.

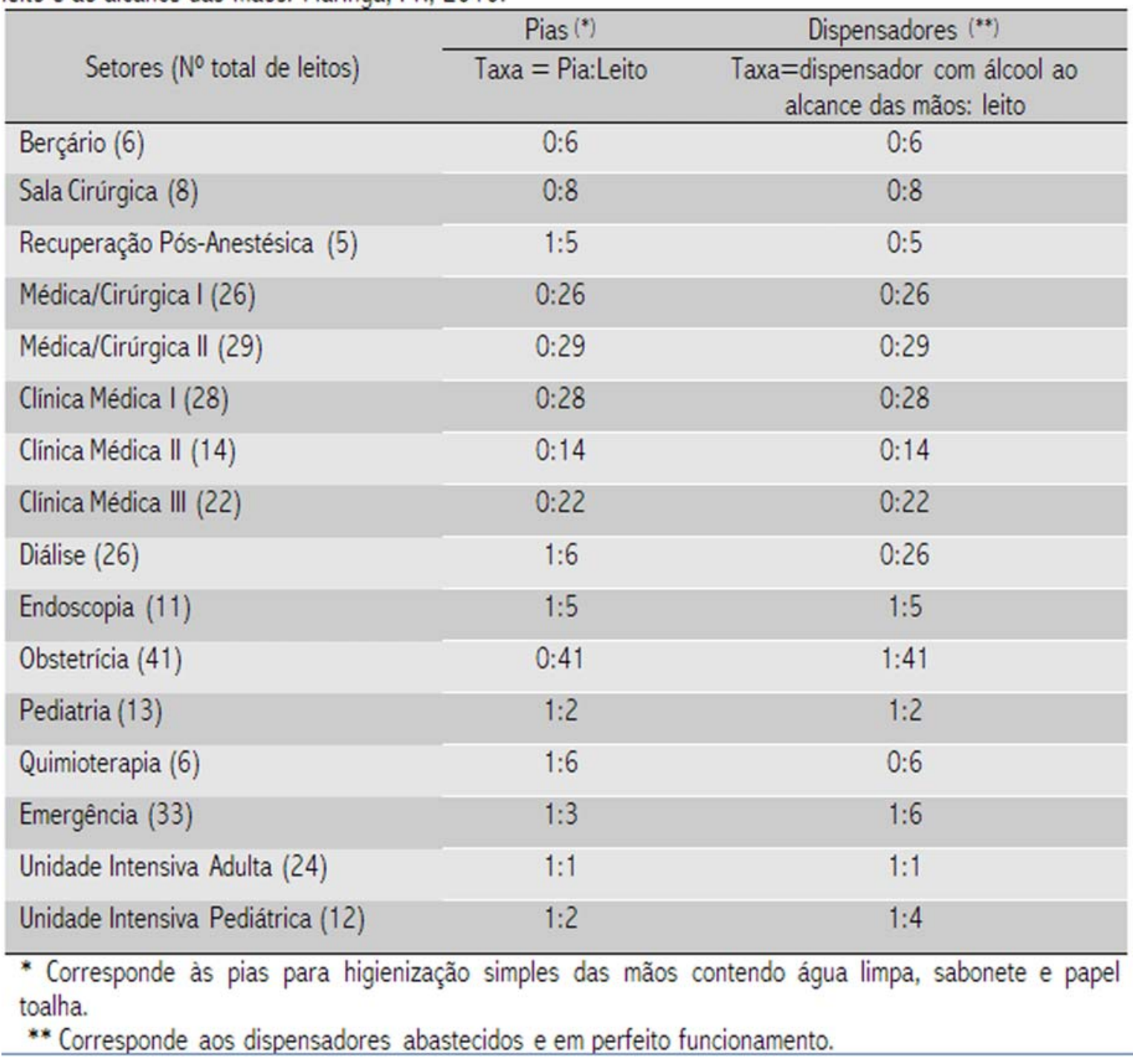

\section{DISCUSSÃO}

Reconhece-se a importância da higienização das mãos para a prevenção e controle das infecções relacionadas à assistência à saúde. Igualmente importante é a estrutura física adequada para a boa prática da higienização das mãos. Entretanto, neste estudo foram identificadas muitas barreiras institucionais que podem comprometer a adesão a esta prática.

Constatou-se que 0 abastecimento dos insumos (sabão líquido e preparaç̃̃es alcoólicas) não era sistemático. Uma investigação similar conduzida em um hospital universitário em Mali também identificou problemas referentes à disponibilidade de insumos e equipamentos, pois somente em $47,4 \%$ das pias, o sabão e o papel toalha estavam disponíveis'.

Verificou-se a predominância dos tipos de dispensadores fixados à parede, contudo, a maioria deles não estava ao alcance das mãos nos pontos de assistência e tratamento. A carência ou inexistência de outros tipos de dispensadores (frascos de bolso, frascos afixados nos carrinhos e bandejas e frascos dispostos à beira leito) também foi observada. 0 dispensador de parede tem a vantagem de estar visível aos profissionais da saúde, pacientes e visitantes e, por estar fixo à parede, facilita a localização pelos profissionais da saúde. Suas desvantagens incluem a ocorrência da instalação distante do ponto de assistência e tratamento e o fato de requerem reabastecimento e manutenção frequentes ${ }^{1}$. Inversamente aos resultados aqui apresentados, os produtos devem estar acessíveis no ponto da assistência e tratamento sem que haja a necessidade de se deixar o local para acessá-los. Normalmente, as preparações alcoólicas para a higienização das mãos portadas pela equipe (frascos de bolso), preparações alcoólicas afixadas próximas ao leito ou à cabeceira do paciente e preparações alcoólicas afixadas em carrinhos ou colocadas em bandeja de curativo ou medicamentos cumprem esta função. Isso permite que a equipe possa aderir, de forma rápida e fácil, as indicações para se higienizar as mãos, que abrange a realização do procedimento antes do contato com o paciente, antes de 
procedimentos limpos e assépticos, após o contato com fluidos corporais, após o contato com o paciente e após o contato com o ambiente próximo ao paciente ${ }^{8}$.

Nos pontos de assistência e tratamento, identificou-se a escassez de cartazes sobre a temática, e, na maioria das unidades, os manuais sobre o tema não estavam disponíveis. É fundamental que lacunas como estas sejam preenchidas por meio de estratégias sustentáveis de promoção à higienização das mãos nos serviços de saúde, o que inclui a instalação de lembretes e cartazes nos pontos de assistência e tratamento que disponham sobre dois elementos essenciais, a técnica de higienização das mãos (como fazer) e sobre os cinco momentos para se higienizar as mãos (quando fazer) ${ }^{9}$. Além disso, a carência de manuais sobre o tema disponível para consulta nas unidades e o desconhecimento dessas diretrizes são relatadas como fatores associados à baixa adesão à higienização das mãos pelos profissionais da saúde?

No que diz respeito aos treinamentos recebidos pelos profissionais da saúde, houve uma disparidade entre as diferentes categorias profissionais, pois os médicos relataram não terem recebido treinamento sobre a temática. Além disso, houve negligência por parte da $\mathrm{CClH}$ em não monitorar a prática da higienização das mãos pelos profissionais da saúde nas unidades. Neste sentido, constata-se o descumprimento institucional em relação à normativa oficial do Ministério da Saúde, pois a Portaria n 2616 publicada em 1998 impõe à CClH a competência de supervisionar a aplicação de normas e rotinas voltadas à prevenção e controle das infecções e ainda recomenda que a instituição deva fomentar a educação e 0 treinamento sobre a prevenção e controle das infecções com todo o pessoal hospitalar ${ }^{10}$. Além disso, aumentar o conhecimento dos profissionais da saúde em relação às boas práticas de higienização das mãos pode colaborar na redução da transmissão das infecções cruzadas ${ }^{11}$.

Incoerentemente, a higienização das mãos foi negligenciada pela $\mathrm{CCIH}$; constata-se esta inércia em relação a um dos elementos fundamentais no combate à infecção relacionada à assistência à saúde pela ausência de cartazes sobre o tema nos locais de assistência e tratamento, ausência de treinamentos sobre a higienização das mãos destinados à categoria dos médicos e ausência de monitoramento das práticas pela CCIH. Negligenciar tal aspecto é negligenciar a assistência de qualidade em saúde, a segurança dos pacientes e os aspectos éticos que envolvem a relação paciente e profissional da saúde e a relação paciente e serviço de saúde. Assim, a ênfase na higienização das mãos deveria ser prioridade da $\mathrm{CClH}$, à medida que necessita incorporar mecanismos de prevenção e controle das infecções para cumprir sua função.

Neste estudo, evidenciou-se a inexistência de pias e dispensadores por leito na maioria das unidades observadas. Nas unidades em que estes equipamentos estavam presentes, a quantidade por leito era excessivamente limitada. A UTI adulto e Pediatria foram exceção, pois apresentaram as maiores taxas que variaram entre 1:1 e 1:2. Similarmente, várias pesquisas mostram a escassez de pias nos hospitais, em que as existentes se localizam fora dos quartos dos pacientese destacam ainda a variação das taxas de pias por leito nos diferentes setores ${ }^{12,13}$. Semelhantemente, a maior taxa de pia por leitos (1:1) também foi identificada em uma UTI ${ }^{13}$. Um estudo de comparação da influência da acessibilidade na adesão à higienização das mãos constatou que a maior taxa de adesão (76\%; 1:1 pia/leito) foi da unidade intensiva médica em relação à unidade intensiva cirúrgica ( $51 \%$ adesão; $1: 4$ pia/leito) ${ }^{4}$. Muitos estudos sugerem que a acessibilidade é um fator importante na adesão à higienização das mãos ${ }^{1,3}$. Entretanto, outros autores afirmam que a acessibilidade aos insumos e equipamentos não aumentam significativamente a taxa de adesão ao procedimento ${ }^{13-15}$, isto porque existe a influência de outras variáveis envolvidas na adesão, tais como pertencer à categoria médica, ser do gênero masculino, usar luvas, esquecimento, sobrecarga de trabalho, número insuficiente de profissionais para a demanda, a falta de exemplos positivos dos colegas de trabalho, a falta de educação permanente, 0 ressecamento da pele pelos produtos e a falta de tempo ${ }^{3}$.

Nessa mesma perspectiva, reconhece-se que adesão à higienização das mãos é multifatorial e complexa. No entanto, neste estudo, ainda se evidencia a negligência dos serviços de saúde em relação os aspectos físicos, pois existem falhas na oferta de insumos e equipamentos, tais como a dificuldade de acesso às pias e dispensadores e localização distante do ponto da assistência ao paciente, o que pode dificultar a adesão. Neste ponto, cumpre refletir sobre a seguinte questão: se os aspectos mais simples, tais como a estrutura física relacionada à higienização das mãos, são negligenciados, como os serviços de saúde estão abordando os demais aspectos mais complexos envolvidos na adesão, tais como os comportamentais? Considera-se extremamente importante a valorização da infraestrutura acessível à higienização das mãos concomitantemente a outras ações institucionais promotoras desta prática, pois o fácil acesso aos insumos e equipamentos pode estimular a higienização das mãos nos serviços de saúde.

Assim, para promover a higienização das mãos, entre as novas recomendações do CDC e OMS está o uso das preparações alcoólicas ${ }^{1,2}$. As vantagens desses produtos incluem a maior eficácia antimicrobiana, menor ressecamento da pele, maior facilidade de uso, menos tempo de ação e a disponibilidade à beira do leito do paciente, facilitando o acesso e estimulando o uso do produto pelos profissionais da saúde ${ }^{9,16}$.

Dada a superioridade das preparações alcoólicas em detrimento aos demais produtos para a higienização das mãos, recentemente a Agência Nacional de Vigilância Sanitária publicou a Resolução da Diretoria Colegiada ${ }^{0}$ 42 que dispõe sobre a obrigatoriedade de disponibilização de preparação alcoólica para fricção antisséptica das mãos, pelos serviços de saúde do país. Estes produtos devem ser 
disponibilizados nos pontos de assistência e tratamento, em lugar visível e de fácil acesso, de forma que os profissionais de saúde não necessitem deixar o local de assistência e tratamento para higienizar as mãos ${ }^{17}$.

Contudo, quanto aos serviços de saúde, não basta atender integralmente às exigências oficiais, pois a promoção da higienização das mãos depende de estratégias multimodais baseadas em, ao menos, cinco componentes, tais como a educação permanente dos profissionais da saúde, a monitorizarão sistemática das práticas relativas a este procedimento e a realimentação deste desempenho aos profissionais da saúde, instalação de lembretes de promoção à higienização das mãos localizados em pontos estratégicos nas unidades, adoção de um clima institucional seguro e recorrer ao uso das preparações alcoólicas como procedimento padrão para a higienização das mãos ${ }^{1,9}$.

Neste estudo, o impacto da estrutura física na taxa de adesão à higienização das mãos não foi mensurado; isto se torna um fator limitante dada a escassez de publicações que evidenciam a relação entre essas variáveis. Além disso, a inexistência de publicações nacionais de avaliação da infraestrutura para a prática da higienização das mãos impossibilitou a comparação dos nossos resultados no cenário nacional.

Sugere-se que estudos similares sejam conduzidos no Brasil, pois conhecer a infraestrutura dos serviços de saúde voltada à prática de higienização das mãos poderá fornecer subsídios aos gestores, profissionais do controle de infecção e profissionais da saúde para superarem os desafios impostos a esta prática.

Resultados positivos voltados ao controle de infecções relacionadas à assistência à saúde são alcançados com 0 trabalho em equipe, o que inclui todos profissionais que prestam os cuidados diretos ao paciente, a gestão, as instalações, a provisão de materiais, equipamentos e produtos ${ }^{18}$.

A transformação da realidade dos serviços de saúde em prol de uma assistência mais limpa e segura inclui a adequação da estrutura física em conformidade com as exigências da normativa oficial vigente e a adoção das estratégias multimodais pelos responsáveis pela $\mathrm{CCIH}$ à luz dos pressupostos norteadores da OMS.

Assim, acredita-se que a ética do cuidado nos serviços de saúde se articula com o reconhecimento da higienização das mãos como prioridade institucional, pois valorizar e assegurar esta prática pode qualificar a assistência em saúde e garantir a segurança do paciente.

\section{CONCLUSÃO}

Nas unidades assistenciais, houve variação na disponibilidade de insumos e equipamentos para a prática da higienização das mãos, pois a água sempre estava disponível, as preparações alcoólicas não estavam disponíveis em todas as unidades e a maioria dos dispensadores disponíveis não era abastecida sistematicamente.

Em relação ao tipo de dispensador, predominou o tipo de dispensador de parede nos pontos de assistência, no entanto, localizado distante do alcance das mãos.

Constatou-se a escassez de cartazes ilustrativos sobre a técnica e sobre a promoção da higienização das mãos, fixados nas unidades. Na maioria das unidades não havia manuais sobre a temática disponíveis para consulta pelos profissionais da saúde.

Referente ao treinamento recebido pelos enfermeiros e médicos sobre a higienização das mãos, nos últimos dois anos, verificou-se a disparidade na participação entre as categorias profissionais, com a predominância dos enfermeiros em detrimento aos médicos. Verificou-se que as auditorias sobre a higienização das mãos não eram realizadas pela $\mathrm{CCIH}$ nas unidades assistenciais.

Houve variação na taxa de pias por leito e taxa de dispensadores por leito nas unidades. Na metade, inexistiam pias nos pontos de assistência. Na maioria das unidades não havia dispensadores nos pontos de assistência.

Conclui-se que 0 abastecimento não sistemático dos insumos, a escassez de pias por leito e a inexistência de dispensadores por leito na maioria das unidades são falhas na infraestrutura voltada à prática da higienização das mãos.

\section{REFERÊNCIAS}

1 World Health Organization. WHO guidelines on hand hygiene in health care. First global patient safety challenge: clean care is safe care. Geneva (SUI): WHO; 2009.

2 Boyce JM; Pittet D. Guideline for Hand Hygiene in Health-Care Settings: Recommendations of the Healthcare Infection Control Practices Advisory Committee and the HICPAC/SHEA/APIC/IDSA Hand Hygiene Task Force. Infect Control Hosp Epidemiol. 2002; 23(12): 3-40.

3 Pittet D. Improving compliance with hand hygiene in hospitals. Infect Control Hosp Epidemiol. 2000; 21: 381-6.

4 Kaplan LM; McGuckin M. Increasing handwashing compliance with more accessible sinks. Infect Control Hosp Epidemiol. 1986; 7(8): 408-10.

5 Boyce JM. Antiseptic Technology: Access, Affordability and Acceptance. Emerg Infect Dis. 2001 mar/apr; 7(2): 231-3.

6 Suresh G, Cahill J. How "user friendly" is the hospital for practicing hand hygiene? An ergonomic evaluation. Jt Comm I Qual Patient Saf. 2007 mar; 33(3): 171-9.

7 Organização Mundial da Saúde. Guia para implementação: um Guia para a implantação da estratégia multimodal da OMS para a melhoria da higienização das mãos a observadores. Brasília (DF): Organização PanAmericana da Saúde; Agência Nacional de Vigilância Sanitária; 2008. 
8 Organização Pan-Americana da Saúde; Agência Nacional de Vigilância Sanitária. Manual para observadores: estratégia multimodal da OMS para a melhoria da higienização das mãos. Brasília (DF): Organização PanAmericana da Saúde; 2008.

9 Pitttet, D. Hand Hygiene: It's All About When and How? Infect Control Hosp Epidemiol. 2008 oct.; 29 (10): 957-9.

10 Portaria n. 2616, de 12 de maio 1998. Dispõe sobre a regulamentação das ações de controle de infecção hospitalar no país. Diário Oficial da União. Brasilia, 13 mai 1998: Seção 1:1

11 Kampf G. State-of-the-art hand hygiene in community medicine. Int J Hyg Environ Health . 2003 oct.; 206(6): 465-72.

12 Basurrah M; Madani TA. Handwashing and gloving practice among health care workers in medical and surgical in a tertiary centre in Riyadh Saudi Arabia. Scand J Infect Dis. 2006; 38(8): 620-4.

13 Vernon MO; Trick WE, Welbel SF; Peterson BJ, Weinstein RA. Adherence with ;hand hygiene: does number of sinks matter? Infect Control Hosp Epidemiol. 2003 mar.; 24(3): 224-5.

14 Lankford MG; Zembower TR; Trick WE; Hacek DM; Noskin GA, Peterson LR. Influence of role models and hospital design on hand hygiene of health care workers. Emerg Infect Dis. 2003 feb.; 9(2): 217-23.

15 Whitby M; McLaws ML. Handwashing in healthcare workers: accessibility of sink location does not improve compliance. J Hosp Infect. 2004 dec.; 58(4): 247-53.

16 Pittet D; HugonnetS; Harbarth S; Mourouga P; Sauvan V; Touveneau S et al. Effectiveness of a hospital-wide programme to improve compliance with hand hygiene. Lancet. 2000 oct.; 356: 1307-12.

17 Ministério da Saúde (Brasil). Agência Nacional de Vigilância Sanitária. Resolução da Diretoria Colegiada n. 42, de 25 de outubro de 2010. Aprova a obrigatoriedade. Diário Oficial União. Brasília, 2010. Seção 1, p. 27-8.

18 Aguiar DF; Lima ABG; Santos RB. Uso das precauções-padrão na assistência de enfermagem: um estudo retrospectivo. Esc. Anna Nery Rev. Enferm. 2008 set.; 12(3): 571-5. 